Old Dominion University

ODU Digital Commons

Bioelectrics Publications

Frank Reidy Research Center for Bioelectrics

2002

\title{
Improved Energy Model for Membrane Electroporation in Biological Cells Subjected to Electrical Pulses
}

\author{
R. P. Joshi \\ Old Dominion University \\ Q. $\mathrm{Hu}$ \\ Old Dominion University \\ K. H. Schoenbach \\ Old Dominion University \\ H. P. Hjalmarson
}

Follow this and additional works at: https://digitalcommons.odu.edu/bioelectrics_pubs

Part of the Cell Biology Commons, Electrical and Electronics Commons, and the Medicine and Health Sciences Commons

\section{Repository Citation}

Joshi, R. P.; Hu, Q.; Schoenbach, K. H.; and Hjalmarson, H. P., "Improved Energy Model for Membrane Electroporation in Biological Cells Subjected to Electrical Pulses" (2002). Bioelectrics Publications. 241.

https://digitalcommons.odu.edu/bioelectrics_pubs/241

\section{Original Publication Citation}

Joshi, R. P., Hu, Q. Schoenbach, K. H., \& Hjalmarson, H. P. (2002). Improved energy model for membrane electroporation in biological cells subjected to electrical pulses. Physical Review E, 65(4), 041920. doi:10.1103/PhysRevE.65.041920

This Article is brought to you for free and open access by the Frank Reidy Research Center for Bioelectrics at ODU Digital Commons. It has been accepted for inclusion in Bioelectrics Publications by an authorized administrator of ODU Digital Commons. For more information, please contact digitalcommons@odu.edu. 


\title{
Improved energy model for membrane electroporation in biological cells subjected to electrical pulses
}

\author{
R. P. Joshi, Q. Hu, and K. H. Schoenbach \\ Department of Electrical and Computer Engineering, Old Dominion University, Norfolk, Virginia 23529-0246
}

\author{
H. P. Hjalmarson
}

Computational Biology and Materials Technology Department, Sandia National Laboratory, Albuquerque, New Mexico 87185-1111

(Received 31 October 2001; revised manuscript received 1 February 2002; published 9 April 2002)

\begin{abstract}
A self-consistent model analysis of electroporation in biological cells has been carried out based on an improved energy model. The simple energy model used in the literature is somewhat incorrect and unphysical for a variety of reasons. Our model for the pore formation energy $E(r)$ includes a dependence on pore population and density. It also allows for variable surface tension, incorporates the effects of finite conductivity on the electrostatic correction term, and is dynamic in nature. Self-consistent calculations, based on a coupled scheme involving the Smoluchowski equation and the improved energy model, are presented. It is shown that $E(r)$ becomes self-adjusting with variations in its magnitude and profile, in response to pore population, and inhibits uncontrolled pore growth and expansion. This theory can be augmented to include pore-pore interactions to move beyond the independent pore picture.
\end{abstract}

DOI: 10.1103/PhysRevE.65.041920

PACS number(s): 87.15.Aa, 87.50.Rr, 87.17.Aa

\section{INTRODUCTION}

Electroporation is a well-known physical process in biological cells [1-3]. It involves rapid structural rearrangement of the membrane, in response to an externally applied electric field. A prominent observable effect is the rapid increase of electrical conductivity [4] attributed to the formation of pores in the lipid bilayer membrane. The opening of such channels (or more appropriately, transient aqueous pores) enables the transport of ions and water-soluble species. Electroporation can, therefore, be used to initiate large molecular fluxes for purposes of introducing genetic material into cells, and numerous applications are beginning to emerge [5-9]. This process has also been linked to the nonthermal killing of microorganisms subjected to strong electric fields [10]. For this reason, it offers great potential for decontamination and the elimination of harmful microorganisms and biohazards.

The exact mechanism for electroporation is still not fully understood, and the mathematical models are inexact and incomplete [11]. We focus here on the inadequacy of the mathematical model, and present appropriate modifications to better represent the inherent physics. Towards this goal, we start with a brief background on electroporation modeling. Litster [12] and Taupin, Dvolaitzky, and Sauterey [13] were the first to suggest the role of thermal fluctuations in pore formation, and the existence of a threshold poreformation energy. The basic model was subsequently extended to include electrostatic effects $[14,15]$. The biophysical description was translated into numerical models [16-18] based on the Smoluchowski equation [19] to predict the evolutionary pore dynamics. Since the pore dynamics is influenced by the transmembrane potential $U(r, t)$ calculations of $U(r, t)$ need to be included for self-consistency. Most studies, with the exception of a short report by Vaughan and Weaver [20], have ignored this aspect. Only very recent simulations by our group have accounted for self-consistency through the use of lumped equivalent circuits [21], or the inclusion of electric field solvers [22].

Predictions of pore generation, growth, and size evolution are based on continuum Smoluchowski theory, with the following governing equation for the pore density distribution function $n(r, t)$ :

$$
\begin{gathered}
\partial n(r, t) / \partial t-\left\{D /\left[k_{B} T\right]\right\}[\partial\{n(r, t) \partial E(r) / \partial r\} / \partial r] \\
-D\left[\partial^{2} n(r, t) / \partial r^{2}\right]=S(r),
\end{gathered}
$$

where $S(r)$ is the source (or pore formation) term, while $D$ is a pore diffusion constant. See Table I for sources and values of parameters. Physically, the diffusion process represents a "random walk" of the pore radius in " $r$ space," brought about by fluctuations in radius arising from the constant entry and egress of water molecules and other species. The formation of pores is generally assumed to be a two-step process [23-25]. All pores are initially created as

TABLE I. Parameters used for the theoretical model.

\begin{tabular}{llc}
\hline \hline \multicolumn{1}{c}{ Parameter } & Source & Value \\
\hline$D\left(\mathrm{~m}^{2} \mathrm{~s}^{-1}\right)$ & Ref. [19] & $5 \times 10^{-14}$ \\
$\gamma\left(\mathrm{J} \mathrm{m}^{-1}\right)$ & Ref. [19] & $1.8 \times 10^{-11}$ \\
$\Gamma_{0}\left(\mathrm{~J} \mathrm{~m}^{-2}\right)$ & Ref. [19] & $10^{-3}$ \\
$C\left(\mathrm{~J}^{1 / 4} \mathrm{~m}\right)$ & Ref. [24] & $9.67 \times 10^{-15}$ \\
$K_{w}\left(\mathrm{~F} \mathrm{~m}^{-1}\right)$ & Refs. [19,26] & $80 \times 8.85 \times 10^{-12}$ \\
$K_{m}\left(\mathrm{~F} \mathrm{~m}^{-1}\right)$ & Refs. [19,26] & $2 \times 8.85 \times 10^{-12}$ \\
$h(\mathrm{~m})$ & Ref. [26] & $5 \times 10^{-9}$ \\
$a_{p}\left(\mathrm{~F} \mathrm{~m}^{-2}\right)$ & Ref. [24] & $6.9 \times 10^{-2}$ \\
$v_{c}\left(\mathrm{~m}^{-3} \mathrm{~s}^{-1}\right)$ & Ref. [14] & $2 \times 10^{38}$ \\
$v_{d}\left(\mathrm{~s}^{-1}\right)$ & Ref. [26] & $10^{11}$ \\
$r_{o}(\mathrm{~m})$ & Ref. [26] & $1 \times 10^{-9}$ \\
\hline \hline
\end{tabular}


hydrophobic/nonconducting, at a rate of $S(r)$ per unit area of the membrane, during every time interval " $d t$. ." This rate is given as

$$
S(r)=\left\{\left(v_{c} h\right) /\left(k_{B} T\right)\right\}[d E(r) / d r] \exp \left[-E(r) /\left(k_{B} T\right)\right] d r,
$$

where $v_{c}$ is an attempt rate density [14], $E(r)$ the energy for hydrophobic pores, $T$ the operating temperature, $k_{B}$ the Boltzmann constant, and $A$ the average membrane area. If a nonconducting pore is created with a radius $r>r^{*}(=0.5$ $\mathrm{nm}$ ), it spontaneously changes its configuration and transforms into a conducting, hydrophilic pore. All conducting pores then survive as long as their radii remain larger than $r^{*}$. Destruction of a conducting pore occurs only if it drifts or diffuses in $r$ space to a value below $r^{*}$. Due to the exponential term in Eq. (2), most pores are created with very small radii.

It is thus clear from Eqs. (1) and (2) that the energy $E(r)$ is the most important entity that governs the pore formation, growth, and decay. This energy $E(r)$, which is a function of the pore radius " $r$," determines the "drift flux" for pores in $r$ space [the left side of Eq. (1)], and the formation rate [through Eq. (2)]. Hence, the theoretical accuracy of predictions can only be as good as the precision and correctness of $E(r)$. Here we outline the accepted model for $E(r)$, and in the process, seek to underscore the inherent deficiency and inadequacies. This energy function depends on several factors, including the membrane tension, the applied voltage and associated stored electrostatic energy, and steric repulsion. The published and accepted model of $E(r)$ for hydrophobic and hydrophilic pores, respectively, is $[4,14,23,26]$

$$
E(r)=2 \pi h r \sigma(\infty)\left[I_{1}\left(r / r_{0}\right) / I_{0}\left(r / r_{0}\right)\right]-\pi a_{p} V^{2} r^{2},
$$

and

$$
E(r)=2 \pi \gamma r-\left[\int_{0}^{r} 2 \pi \Gamma\left(r^{*}\right) r^{*} d r^{*}\right]+(C / r)^{4}-\pi a_{p} V^{2} r^{2} .
$$

In the above equations, $I_{1}$ and $I_{0}$ are the modified Bessel functions of the zeroth and first order, respectively, $h$ is the membrane thickness, $\sigma(\infty)$ is a constant on the order of 5 $\times 10^{-2} \mathrm{~N} \mathrm{~m}^{-1}$ [26], while $r_{0}$ represents a characteristic length scale over which the properties of water change between the interface and the bulk. The value of $r_{0}$ is taken to be equal to $1 \mathrm{~nm}[24]$. The $(C / r)^{4}$ term in Eq. (3b) accounts for steric repulsion between the lipid heads lining the pore, and contributes to an increase in energy with a shrinking radius $[3,24]$. A typical value for $C$ has been reported to be about $9.67 \times 10^{-15} \mathrm{~J}^{0.25} \mathrm{~m}$ [24]. The last term in Eq. (3b) represents the capacitive contribution to the energy in the presence of a transmembrane potential " $V$." The coefficient $a_{p}$ is a property of the membrane and its aqueous environment. In the simplest continuum approximation [26], it is expressed in terms of the membrane thickness " $h$ " and the permittivities " $\varepsilon_{w}$ " and " $\varepsilon_{m}$ " of water and the membrane, respectively, as $a_{p}=\left(\varepsilon_{w}-\varepsilon_{m}\right) /[2 h]$. It might be mentioned that other models that take into account pore conductivity and ionic distor-

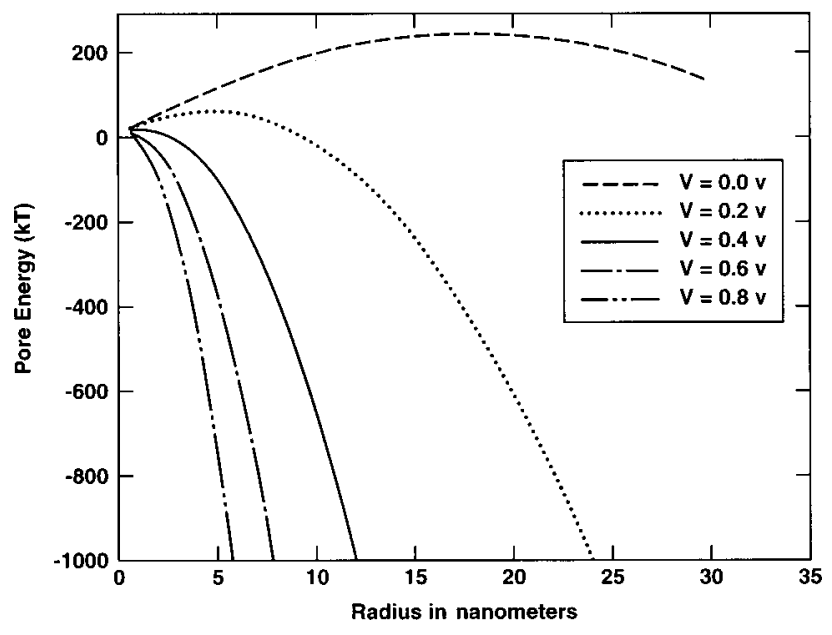

FIG. 1. The pore formation energy $E(r)$ of hydrophilic pores for various membrane voltages.

tions of the electric field $[15,27]$ have been proposed for the electrostatic energy calculations. Finally, $\gamma$ is the energy per unit length of the pore perimeter, while $\Gamma$ is the energy per unit area of the intact membrane.

Most analyses in the literature [24] use a constant surface tension parameter $\left(\Gamma=\Gamma_{0}\right)$, yielding the following simplified formation energy expression for conducting pores:

$$
E(r)=2 \pi \gamma r-\pi \Gamma_{0}+(C / r)^{4}-\left\{\left(\varepsilon_{w}-\varepsilon_{m}\right) /[2 h]\right\} \pi r^{2} V^{2} .
$$

The incorrectness and physical inadequacies of Eq. (4) are best elucidated by considering the predicted voltagedependent behavior. Plots of $E(r)$ based on Eq. (4) are given in Fig. 1. For zero applied voltage, a local minima in the pore energy is predicted at about $0.8 \mathrm{~nm}$. This corresponds to the most likely pore size, under steady-state equilibrium conditions. Figure 1 also predicts a local maxima for the zero volt case, at a pore radius of about $18 \mathrm{~nm}$. From the shape of the energy function it becomes clear that all pores, having radii less than $18 \mathrm{~nm}$, would tend to drift towards smaller values in $r$ space. Physically, the monotonic increase in pore energy below $18 \mathrm{~nm}$ would force pores to shrink in size, and approach the dynamically stable radius of $0.8 \mathrm{~nm}$. However, pores with radii exceeding this threshold, would drift towards larger values and expand without bound in an uncontrolled fashion. Irreversible breakdown and cell rupture would, therefore, be the predicted result, for pores exceeding the stability threshold radius $r_{\text {crit }}$ of $18 \mathrm{~nm}$. In Fig. 1, both the peak energy and radius of the local maxima shift for a $0.2 \mathrm{~V}$ transmembrane potential. The critical radius for stability reduces to about $5.8 \mathrm{~nm}$. In any case, a potential barrier is still seen to exist for the $0.2 \mathrm{~V}$ voltage. However, for $0.4 \mathrm{~V}$ across the cellular membrane, the maxima is virtually eliminated. This, therefore, represents the minimum voltage that would lead to cellular breakdown under this model, provided the voltage was applied long enough to enable pores to grow beyond the 18-nm critical threshold. This model predicts full cell recovery only if the applied voltage was terminated in time before the critical expansion could take place [21]. At 
the higher voltages of 0.6 and $0.8 \mathrm{~V}$, the local maxima is not seen, and the pores can potentially expand irreversibly without bound.

The simple energy model of Eq. (4) is, therefore, incorrect and unphysical for the following reasons.

(i) First, as evident from Fig. 1, there is no barrier for $V>0.4 \mathrm{~V}$. However, from experimental data, much higher membrane voltages of about $1.0 \mathrm{~V}$ are required [28] for irreversible breakdown and membrane rupture.

(ii) Next, the simple calculation for $a_{p}$ in Eq. (4) does not take into account the finite conductivity of pores, or any charge screening effects. Instead, the capacitor model simply replaces the lipid inside a pore with nonconducting water. For a more realistic representation, the transport of ions from a region of high dielectric constant (water) in the proximity of a low dielectric constant layer (lipid) needs to be taken into account, and the energy expenditure $[15,29,30]$ of the process included. Formalisms that model such variations of Born energy have been proposed $[15,18]$.

(iii) The use of a constant surface tension $\Gamma_{0}$ becomes questionable as well. The mechanical properties of cells are expected to be modified by deformation, and changes in membrane area caused by the Maxwell stress tensor associated with an externally applied voltage. Though direct experimental verification of surface tension and its variation is unavailable, molecular dynamics simulations of lipid bilayers do demonstrate the following [31]. (a) A finite tension is required to maintain a given cellular shape and size, and (b) the tension must change with the system area. Also, indirect experimental evidence indicative of variations in membrane tension is available. For example, activation of the 3-ns MscL channel cloned from Escherichia coli [32] has been linked to the tension of lipid membranes. Similarly, the activity of lytic peptides is affected by the tension of vesicles under stress [33], and the catalytic activity of a $\beta$ isoform of phospholipase $C$ shown to change with surface pressure [34]. These experimental results suggest that the tension must naturally be variable, and that its variation facilitates biological activities that are observed. (c) Third, since tension is proportional to the membrane area, at least to first order, it follows that pore formation will lead to variations in $\Gamma$ that are proportional to the square of the pore radius. In order to account for this variability, a simple heuristic model has recently been proposed [35] that describes the tension as $\Gamma(r)=\Gamma_{0}\left[1-r^{2} / r_{\alpha}^{2}\right]$, with $r_{\alpha}$ being a constant parameter. Hence, it follows that pore formation and growth will lead to reduction in the $\Gamma$ parameter. The primary effect of such variations in $\Gamma$, would be the creation of an additional local minima in the pore energy function $E(r)$ which would force the pores to stabilize at some large radius instead of expanding indefinitely. However, it is important to note that the parameter $r_{\alpha}$ of the heuristic model [35] should not be taken to be a constant, but should instead be a time and/or voltagedependent variable to include dynamic effects.

(iv) The formation energy $E(r)$ in Eq. (4) is independent of the pore population and density. However, since the lipid bilayer is essentially elastic and incompressible, it follows that changes in the pore area at constant surfactant molecules, must cause changes in the interfacial free energy.
This was first discussed many years ago by White [36], who also argued in favor of decreases in surface tension with pore growth. The increased interdigitation of the alkyl chains was invoked as the physical mechanism for changes in both the free energy and $\Gamma$. Dependence of the collective pore area, and possible pore-pore interaction effects, on the parameters of Eq. (4) needs to be taken into account. The independent pore model generally assumed will be inadequate as the pore population increases.

(v) Finally, the parameters of Eq. (4) are static, and there is no dependence on the dynamical evolution of the pores. Based on the above argument, not only should $E(r)$ depend on the pore density " $n$," but the magnitude and profile must vary with time in accordance to $n(r, t)$. Such a mechanism would make $E(r)$ self-adjusting in response to $n(r, t)$, without causing uncontrolled pore growth and expansion. Very simply, decreases in surface tension due to pore formation, would increase the cost of creating pore following the trend of Eq. (4). This would potentially work to halt further growth and stabilize the pore population.

The most direct evidence of an inadequacy of the independent pore, constant tension model with its energy maxima at around $18 \mathrm{~nm}$ comes from experimental measurements. For instance, pores with stable diameters up to micrometers in size have been reported $[37,38]$. This observation is clearly contrary to the theoretical prediction of either complete pore closure or unbounded expansion leading to rupture. Similarly, stabilization of pore radii within the 2060-nm range have been reported by Chang and Reese [39] in their studies of red blood cells. The resolution of their experiments allowed the pores to be seen $3 \mathrm{~ms}$ after an applied voltage pulse, when their radii were $10-20 \mathrm{~nm}$. The pores continued to grow, but then stabilized at around 20-60 nm after $40 \mathrm{~ms}$. Given such time-resolved experimental data, it becomes clear that the simple electroporative-energy model needs to be modified to yield better predictions and more accurate, physical results. An attempt towards this goal is discussed in this contribution. The model developed here allows for a variable surface tension, incorporates the effects of finite conductivity on the electrostatic correction term, and is dynamic in nature through a dependence on both the cell voltage and pore density. These changes make $E(r)$ selfadjusting in response to pore formation, without causing uncontrolled growth and expansion. It may also be pointed out that though a few recent studies have presented an inclusion of a coupling between membrane tension and pore area $[35,38,40]$, these analyses were either limited to one giant pore or to a population of pores with identical radii. Also, changes associated with finite conductivity and the dynamic nature had been ignored.

\section{IMPROVED MODEL}

Equation (4) is modified here to include a dynamical aspect and a dependence on the pore population density into $E(r)$. Furthermore, voltage-dependent Born energy corrections arising from the presence of ions in water near pores, as suggested by Pastushenko and Chhizmadzhev [15] and Barnett and Weaver [17], have been incorporated. The electro- 
static contribution $E_{\mathrm{ES}}(r)$ to the formation energy then becomes

$$
E_{\mathrm{ES}}(r)=-\pi\left\{\left(\varepsilon_{w}-\varepsilon_{m}\right) /[2 h]\right\} V^{2} \int_{0}^{r} \alpha^{2}\left(r^{\prime \prime}\right) r^{\prime \prime} d r^{\prime \prime},
$$

where $\alpha(r)=\left[1+\pi r k_{P}(r) /\left\{2 h k_{B}\right\}\right]^{-1}, k_{B}$ is the bulk electrolyte conductivity, $k_{P}(r)$ the conductivity in a pore of radius " $r$." The bulk conductivity $k_{B}$ is given in terms of the electronic charge $q\left(=1.6 \times 10^{-19} \mathrm{C}\right)$, concentration $c_{i}$, the mobility $\mu_{i}$ of the $i$ th ion, and its charged state $Z_{i}$ as $k_{B}$ $=\Sigma_{i}\left(q Z_{i}\right)^{2} \mu_{i} c_{i}$. Similarly, the conductivity $k_{P}(r)$ is roughly given as $[15,18]$

$$
\begin{aligned}
k_{p}(r) \sim & \sum_{i}\left(q Z_{i}\right)^{2} \mu_{i} c_{i} H_{i}(r) \\
& \times \exp \left[P\left\{\varepsilon_{m} \varepsilon_{w}\right\}\left(q Z_{i}\right)^{2} /\left\{4 k_{B} T \pi r \varepsilon_{m}\right\}\right],
\end{aligned}
$$

where $k_{B}=1.38 \times 10^{-23} \mathrm{~J} / \mathrm{K}$ is the Boltzmann constant, $H_{i}(r)$ the steric hinderance factor, and with $P\left(\varepsilon_{m} / \varepsilon_{w}\right)$ being the function described by Parsegian [29]. The factor $H_{i}(r)$ has been given by Renkin [41] in terms of $r_{i}$, the radius of the $i$ th ion, as

$$
\begin{aligned}
H_{i}(r)= & \left\{1-\left(r_{i} / r\right)^{2}\right\}\left[1-2.1\left(r_{i} / r\right)+2.09\left(r_{i} / r\right)^{3}\right. \\
& \left.-0.95\left(r_{i} / r\right)^{5}\right] .
\end{aligned}
$$

Hence, when the pores are all small, the $\alpha$ term in Eq. (5) goes to unity (i.e., in the $r \rightarrow 0$ limit), while $\alpha \rightarrow 0$ in the opposite limit of large pore radius $r$. Physically, this implies that the usual electrostatic energy factor is valid for small pore populations when the radii are also small. However, as the pores begin to grow, the $\alpha$ term and hence the contribution to the energy $E(r)$, begin to decrease in magnitude. In terms of Fig. 1, this translates into a flattening of the $E(r)$ curve beyond the potential barrier in the presence of externally applied voltages.

Next, a pore-density-dependent correction to the surface tension parameter $\Gamma$ is discussed. Considering a lipid bilayer of total area " $A$ " consisting of $2 M$ lipid molecules, the total interfacial energy " $W$ " in the absence of any pores is given as [42].

$$
W=2 M \eta=2 M\left[\sigma^{\prime} a+K / a\right] \cong 2\left[A \sigma^{\prime}+K M^{2} / A\right],
$$

where $\sigma^{\prime}$ is the interfacial energy per area of the hydrocarbon-water interface $\left(\sim 2 \times 10^{-2} \mathrm{~J} \mathrm{~m}^{-2}\right)$, " $a$ " is the area per lipid head, and " $K$ " a constant [42]. Equilibrium is determined by the minima of the energy $W$, and hence, is given by the condition $\partial W / \partial A=0$. This yields a minimum value $W_{0}=4 \sigma^{\prime} A_{0}$ and $K=\sigma^{\prime}\left[A_{0} / M\right]^{2}$, where $A_{0}$ is the equilibrium area for corresponding to $W_{0}$. In general, however, for a total area $A$ different from the equilibrium level $A_{0}$, the energy $W$ can be expressed as $W(A)=2 \sigma^{\prime}[A$ $\left.+A_{0}^{2} / A\right]$. The surface tension $\Gamma_{\text {eff }}$ can effectively be defined in terms of the energy differential since the energy is given as $4 \sigma^{\prime} A_{0}+\int_{A_{0}}^{A} \Gamma_{\text {eff }}\left(A^{\prime}\right) d A^{\prime}=W(A)$. Hence, $\partial W / \partial A=\Gamma_{\text {eff }}(A)$ $=2 \sigma^{\prime}\left[1-\left(A_{0} / A\right)^{2}\right]$, and the effective tension is zero when the lipid bilayer area exactly equals the equilibrium value of $A_{0}$. Usually, the area $A$ slightly exceeds the equilibrium level $A_{0}$. Roughly $A / A_{0} \sim 1.0125$ since this ratio yields a tension of $10^{-3} \mathrm{~J} \mathrm{~m}^{-2}$, a value that has been used in the literature.

Upon the formation of pores of total area $A_{p}$, the total area $A$ remains the same. However, the effective membrane area sections reduces to $A_{M}$ where $A_{M}=A-A_{P}$. Consequently, the expressions of the energy $W\left(A_{M}\right)$ and the tension $\Gamma_{\text {eff }}$ change according to

$$
\begin{aligned}
W\left(A_{M}\right) & =4 \sigma^{\prime} A_{0}+\int_{A_{o}}^{A_{M}} \Gamma_{\mathrm{eff}}\left(A^{\prime}\right) d A^{\prime} \\
& =2 \sigma^{\prime}\left[A-A_{P}+A_{0}^{2} /\left(A-A_{P}\right)\right], \\
\text { and } \Gamma_{\text {eff }}\left(A_{M}\right) & =\partial\left[2 \sigma^{\prime}\left\{A-A_{P}+A_{0}^{2} /\left(A-A_{P}\right)\right\}\right] / \partial A \\
& =2 \sigma^{\prime}\left[1-\left\{A_{0} /\left(A-A_{P}\right)\right\}^{2}\right] .
\end{aligned}
$$

The effective tension in the presence of pores can, therefore, be expressed in terms of the value without pores as

$$
\begin{aligned}
\Gamma_{\mathrm{eff}}\left(A_{P}\right)= & \Gamma_{\mathrm{eff}}\left(A_{P}=0\right) \\
& \times\left[1-\left\{A_{0} /\left(A-A_{P}\right)\right\}^{2}\right] /\left[1-\left\{A_{0} / A\right\}^{2}\right] .
\end{aligned}
$$

It follows from Eq. (8c) that the effective tension can be positive, zero or even negative. The zero level corresponds to a situation where the pore area $A_{P}=A-A_{0}$. For higher pore areas (i.e., larger average pores), the $\Gamma_{\text {eff }}$ value can be negative as the membrane is under compression. Finally, the pore area in the above analysis represents the average value and hence, is given in terms of the actual pore density distribution function $n(r, t)$ as

$$
A_{P}(r, t) \sim A_{0}\left[\int_{0}^{r} 2 \pi r^{*} n\left(r^{*}, t\right) d r^{*}\right],
$$

provided mutual pore coupling and pore-pore interactions are negligible. Obviously, the pore density function $n(r, t)$ can be time dependent and as controlled by the Smoluchowski equation for pore growth, drift, and diffusion in $r$ space. Furthermore, the $A_{P}(r, t)$-dependent variable surface tension can become quite important for situations involving transient voltage pulses. In such cases, the voltage could fall to zero quickly, thereby, canceling out the electrostatic contribution to $E(r)$. However, the $A_{P}(r, t)$ term would continue to affect dynamical evolution over much longer periods.

Putting all of the above factors together, the pore formation energy $E(r, t)$ can comprehensively be expressed in terms of the following equation:

$$
\begin{aligned}
E(r)= & 2 \pi \gamma r-\left\{\int_{0}^{r} 2 \pi \Gamma_{\text {eff }}\left(A_{P}\left[r^{*}, t\right]\right) r^{*} d r^{*}\right\}+(C / r)^{4} \\
& -\pi\left\{\left(\varepsilon_{w}-\varepsilon_{m}\right) / h\right\} V^{2} \int_{0}^{r} \alpha^{2}\left(r^{\prime \prime}\right) r^{\prime \prime} d r^{\prime \prime}
\end{aligned}
$$




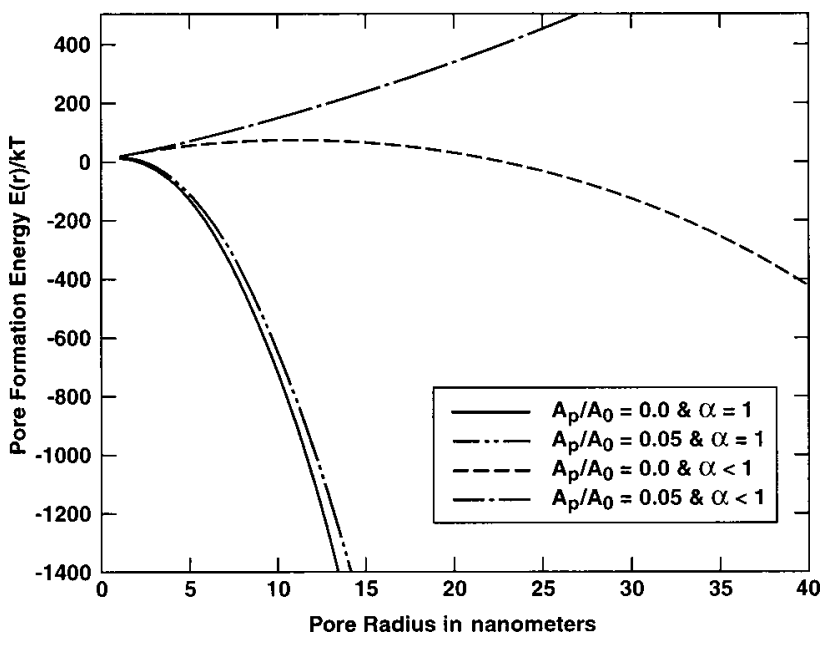

FIG. 2. Pore formation energy function for a $0.4 \mathrm{~V}$ transmembrane bias under various conditions of surface tension and pore population.

with $A_{P}\left[r^{*}, t\right]$ changing dynamically as dictated by Eqs. (1) and $(8 \mathrm{~d})$.

\section{RESULTS AND DISCUSSION}

While a self-consistent solution of the coupled equations (1), (8d), and (9) is necessary, we first present some simple results based on Eq. (9) alone for fixed $A_{P}$ values. The motivation for these calculations was simply to demonstrate the changes in $E(r)$ produced by the modified model, and to facilitate relevant comparisons with the results of Fig. 1. Though strictly a constant $A_{P}$ assignment is inaccurate because of the dynamic nature of the system, its use nonetheless helps us to provide physical insights of pore diffusion in $r$ space and afford qualitative trends of the $n(r, t)$ evolution at a specific time instant. Figure 2 shows $E(r)$ vs $r$ with and without the improved electrostatic correction term (i.e., $\alpha$ $<1$ and $\alpha=1$, respectively). The calculations included two cases: one with no pores $\left(A_{P} / A_{0}=0\right)$ and the other with a specific pore area given by $A_{P} / A_{0}=0.05$. The membrane voltage for Fig. 2 was set at $0.4 \mathrm{~V}$. With $\alpha=1$, the voltagedependent contribution to the pore formation energy is quite dominant, and leads to large negative $E(r)$ values with a monotonically increasing slope for larger radii. Also, there is no potential barrier, and this trend is predicted both with and without pores. Due to the pore-dependent correction in surface tension [via Eq. (8c)], the curve with $A_{P} / A_{0}=0.05$ is slightly higher. Upon including the role of finite ionic conductivity in the pores through an $\alpha(r)<1$ term [as given in Eq. (5)], the pore formation energy is seen to increase dramatically. A local maxima corresponding to a slight potential barrier is evident in Fig. 2 at a radius of about $13 \mathrm{~nm}$ for the $\alpha<1$ and $A_{P} / A_{0}=0.05$ case. Including the surface tension correction as well for $\alpha<1$ completely changes the energy function. Instead of a convex curve, $E(r)$ becomes slightly concave with positive values throughout the entire $0-40 \mathrm{~nm}$ radial range. Physically, this implies that the system would naturally drive the pores towards lower radii (and hence, smaller $\left.A_{P} / A_{0}\right)$ under these conditions. Alternatively, a pore

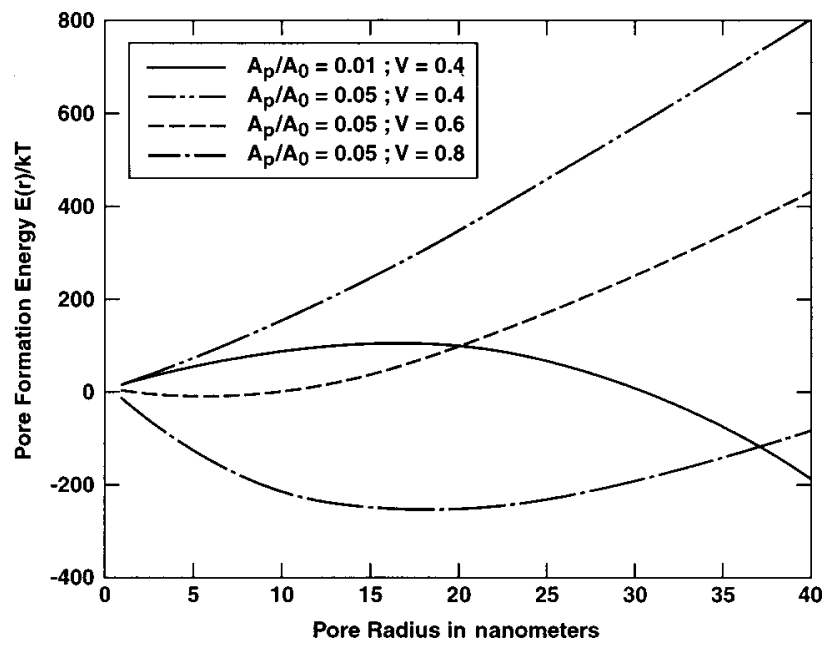

FIG. 3. Dependence of $E(r)$ on membrane voltage and relative pore population.

population of such large radii (effectively leading to $A_{P} / A_{0}$ $=0.05$ ) would not be created or supported in the first place, at this $0.4 \mathrm{~V}$ bias. It also becomes apparent that the effect of having a finite pore population (i.e., $A_{P}>0$ ) is stronger when the ionic conduction term (i.e., $\alpha<1$ ) is also taken into account.

Similarly, the behavior of $E(r)$ on membrane voltage and relative pore population, but without the dynamic, selfconsistent calculations involving $n(r, t)$, is shown in Fig. 3. At the lowest membrane bias of $0.4 \mathrm{~V}$ and a relatively high $A_{P} / A_{0}$ ratio of 0.05 , the $E(r)$ curve is positive and exhibits a monotonic increase with radius. For a slightly lower value of $A_{P} / A_{0}=0.01$ at $0.4 \mathrm{~V}$ (correspondingly also to a lower surface tension), the curve is dramatically altered and exhibits a local maxima at $r \sim 16.5 \mathrm{~nm}$, with negative $E(r)$ values beyond $31 \mathrm{~nm}$. Thus, there is a shift from an unconditionally stable situation for $A_{P} / A_{0}=0.05$, to potential instability with a change in the pore population. The curve for a $0.6 \mathrm{~V} \mathrm{mem-}$ brane potential and $A_{P} / A_{0}=0.05$ exhibits a concave structure with a clear energy minima at around $7 \mathrm{~nm}$. Thus, under these conditions, the cell is predicted to remain perforated in a stable manner without irreversible rupture. Finally, at a still higher bias of $0.8 \mathrm{~V}$, the trend remains unaltered, though the location of the stable minima is predicted to shift to the higher radial value of $18 \mathrm{~nm}$. The central point that becomes transparent from the curves of Figs. 2 and 3 is that the stability of the porated cell is delicately controlled by a combination of parameters that include surface tension, the ion conductance, and pore population. Furthermore, the modified energy model predicts that changes in the magnitude and slope of $E(r)$ can easily occur to profoundly impact the diffusion of pores in $r$ space. Finally, a self-adjustment in $E(r)$ arising from changes in $n(r, t)$ [and hence, $A_{P} / A_{0}$ ] would make it possible to curb uncontrolled pore growth and expansion.

Self-consistent calculations were performed next by coupling the Smoluchowski equation with Eq. (9) for the pore formation energy. A $1.5 \mathrm{~V}, 1 \mu \mathrm{s}$ external pulse was assumed for the analysis. For purposes of quantifying the role of a 


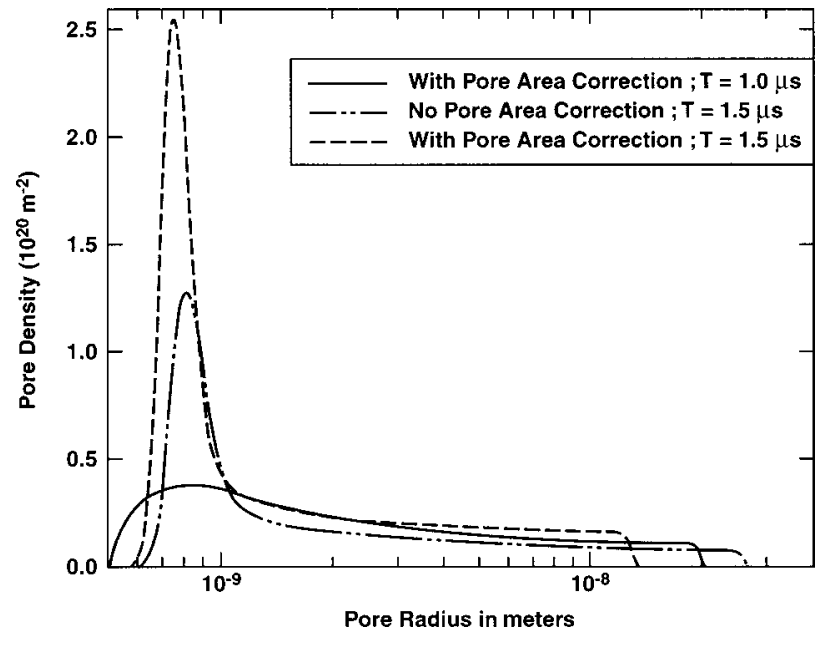

FIG. 4. Calculated pore distribution $n(r)$ at time instants of 1.0 and $1.5 \mu$ s in response to a $1.0 \mathrm{~V}, 1 \mu$ s electrical pulse. Curves with and without the pore area dependence in the energy model are shown.

pore area on the dynamic evolution, two sets of simulations were carried out. In one, a constant surface tension was used [i.e., $\left.\Gamma_{\text {eff }}\left(A_{P}=0\right)\right]$, while for the other simulation set, a porearea-dependent formation energy as given by Eqs. (8c) and (9) were utilized. Results of the pore density distributions $n(r)$ for both simulation conditions are shown in Fig. 4 at the specific time instants of 1.0 and $1.5 \mu \mathrm{s}$. Comparison of the two 1.5 - $\mu$ s curves (with and without the areal correction, i.e., $A_{P} \neq 0$ and $A_{P}=0$, respectively) of Fig. 4, brings out the following features.

(i) A stronger peak with inclusion of the pore area term that roughly lies at a radius of $0.77 \mathrm{~nm}$. Without the pore area term, on the other hand, the most probable radius is predicted to be somewhat larger at $0.82 \mathrm{~nm}$.

(ii) Without the pore area term, the $n(r)$ distribution is predicted to have a much larger spread with pore radii as large as $27 \mathrm{~nm}$. With $A_{P} \neq 0$, the maximum pore radius after $1.5 \mu \mathrm{s}$ is predicted from Fig. 4 to be only about $13 \mathrm{~nm}$. These results can easily be understood in terms of a higher pore formation energy (as shown qualitatively in the curves of Fig. 2) for $A_{P} \neq 0$, and the positive slope that leads to a diffusion in $r$ space towards smaller radii. Thus, the overall result is a faster recovery upon the inclusion of the poredependent (and hence, variable surface tension) factor. The 1.0- $\mu$ s curve for $A_{P} \neq 0$ is flatter than the corresponding $1.5-\mu$ s curve with a larger variance and higher peak pore radius, as might be expected. With the voltage pulse just at the point of being turned off, the pore distribution is out of equilibrium, but begins its shift towards a low-profile, equilibrium profile.

The effects of including the pore-dependent formation energy $E(r)$ are also made evident through the time dependence of the average pore radius $\langle R(t)\rangle$. Plots of $\langle R(t)\rangle$ up to a $1.5 \mu$ s time, with and without the pore area factor, are shown in Fig. 5. Both curves increase monotonically as long as the $1-\mu$ s voltage pulse remains effective. However, the growth of pores is not quite as rapid for $A_{P} \neq 0$, and hence, the average radius does not increase quite as much. Beyond 1

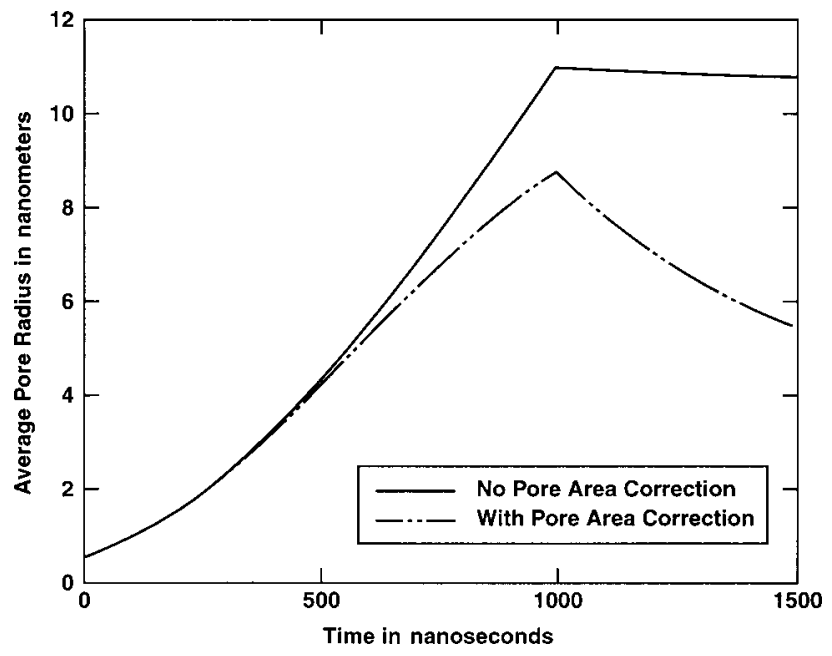

FIG. 5. Results of the average pore radius $\langle R(t)\rangle$ to demonstrate the effect of including a pore dependent energy model.

$\mu$, both curves begin to decrease as the pores begin to shrink. However, for $A_{P} \neq 0$, there is a driving force towards smaller radii for pores of all sizes as governed by a positive slope for the $E(r)$ function. Consequently, the average size decreases at a fairly rapid rate. However, in the absence of a pore area term, the $E(r)$ function has a local maxima at about $18 \mathrm{~nm}$ as given by the $V=0$ curve of Fig. 1. Consequently, pores with radii below 18-nm shrink, while those above $18 \mathrm{~nm}$ continue to grow. The two almost offset each other, and only a small net decrease in $\langle R(t)\rangle$ is predicted in Fig. 5. The time evolution of the pore formation energy $E(r, t)$ that dictates the dynamics and movement in $r$ space, is shown in Fig. 6. With no pore corrections, $E(r)$ at $1.5 \mu \mathrm{s}$ exhibits a slight maxima, and has both positive and negative slopes. With $A_{P}$ taken into account, a concave curve with a positive slope is seen for both the 1.0 and $1.5 \mu$ s time instants. The 1.0- $\mu$ s curve is slightly higher because of the higher pore area at this earlier time. As the system tends towards equilibrium and pores shrink, the pore area de-

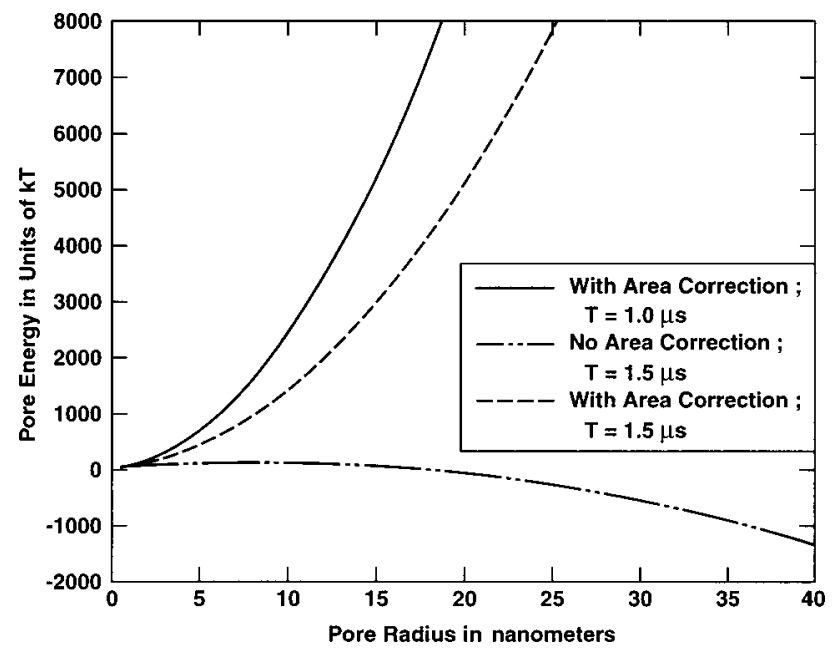

FIG. 6. Pore formation energy $E(r, t)$ under three conditions showing its dynamic nature. 
creases and leads to a lowering of the $E(r)$ curve. The dynamic feature of $E(r, t)$ is thus made obvious.

\section{SUMMARY AND CONCLUSIONS}

A self-consistent model analysis of electroporation in biological cells has been carried out based on an improved energy model. The simple energy model used in the literature appears to be somewhat incorrect and unphysical for a variety of reasons. For example, it predicts cell instability and incessant pore expansion upon the application of external voltages, does not take into account the finite conductivity of pores, or any charge screening effects. Besides the use of a constant surface tension $\Gamma_{0}$ seems to become questionable in light of several experimental reports. For instance, pores with stable diameters up to micrometers in size have been reported $[37,38]$. This observation is in contrast to the theoretical prediction of either complete pore closure or unbounded expansion leading to rupture by the simple prevalent model. Similarly, stabilization of pore radii within the 20-60-nm range have been reported by Chang and Reese [39] in their studies of red blood cells, which are also not predicted by the simple theory.

Here we present an improved model that includes a dependence of pore population and density on the pore formation energy. It also allows for a variable surface tension, and incorporates the effects of finite conductivity on the electrostatic correction term. Finally, the model is dynamic in nature, through its dependence on both the cell voltage and pore density. It has been shown that this will lead to temporal variations in the magnitude and profile of $E(r)$. Such a mechanism would make $E(r)$ self-adjusting in response to pore formation, without causing uncontrolled growth and expansion. Self-consistent calculations based on a coupled scheme involving the Smoluchowski equation and the improved energy model has been carried out. Our results demonstrate the effects of external electrical voltages on the pore dynamics. In principle, this theory can be augmented to include pore-pore interactions to move beyond the independent pore picture. It must also be mentioned that the actual membrane potential is more likely to be the sum of exponentials. This would inherently arise from the "charging" and "discharging" phenomena associated with the inductive and capacitive elements inherent to the cell suspension and the external circuitry. Such circuit and distributed effects were ignored in the present contribution, as the intent was simply to present an improved fundamental model for the energy function $E(r)$. However, these issues can easily be included as shown previously by our group in a related context $[21,22]$.

\section{ACKNOWLEDGMENTS}

The authors would like to thank J. Weaver (MIT), W. Krassowska (Duke University), and L. Douglas Frink (Sandia National Laboratory) for useful discussions. This work was sponsored in part by the Air Force Office of Scientific Research.
[1] R. Stampfli, An. Acad. Bras. Cienc. 30, 57 (1958).

[2] T. Y. Tsong, Biophys. J. 60, 297 (1991).

[3] J. C. Weaver and Yu. A. Chizmadzhev, Bioelectrochem. Bioenerg. 41, 135 (1996).

[4] I. G. Abidor, V. B. Arakelyan, L. V. Chernomordik, Y. A. Chizmadzhev, V. F. Pastushenko, and M. R. Tarasevich, Bioelectrochem. Bioenerg. 6, 37 (1979).

[5] G. A. Hoffmann, S. B. Dev, and G. S. Nanda, IEEE Trans. Biomed. Eng. 46, 752 (1999).

[6] D. C. Chang, B. M. Chassy, J. A. Saunders, and A. E. Sowers, Guide to Electroporation and Electrofusion (Academic, New York, 1992).

[7] E. Neumann, E. Sowers, and C. A. Jordan, Electroporation and Electrofusion in Cell Biology (Plenum, New York, 1989).

[8] S. Orlowski and L. M. Mir, Biochim. Biophys. Acta 1154, 51 (1993).

[9] J. C. Weaver, J. Cell. Biochem. 51, 426 (1993).

[10] A. J. H. Sale and W. A. Hamilton, Biochim. Biophys. Acta 148, 781 (1967).

[11] H. Huelsheger, J. Potel, and E. G. Niemann, Radiat. Environ. Biophys. 20, 53 (1981).

[12] J. D. Litster, Phys. Lett. 53A, 193 (1975).

[13] C. Taupin, M. Dvolaitzky, and C. Sauterey, Biochim. 14, 4771 (1975).

[14] J. C. Weaver and R. A. Mintzer, Phys. Lett. 86A, 57 (1981).

[15] V. F. Pastushenko and Yu. A. Chhizmadzhev, Biofizika 28,
1036 (1983); V. F. Pastushenko and Yu A. Chhizmadzhev, Biofizika 27, 475 (1982).

[16] V. F. Pastushenko, Yu. A. Chhizmadzhev, and V. B. Arakelyan, Bioelectrochem. Bioenerg. 6, 53 (1979).

[17] A. Barnett and J. C. Weaver, Bioelectrochem. Bioenerg. 25, 163 (1991).

[18] S. A. Freeman, M. A. Wang, and J. C. Weaver, Biophys. J. 67, 42 (1994).

[19] For example, R. K. Pathria, Statistical Mechanics (Pergamon, Oxford, 1972), pp. 451-455.

[20] T. Vaughan and J. C. Weaver, in Electricity and Magnetism in Biology and Medicine, edited by A. Bersani (Kluwer Academic, New York, 1999), p. 433.

[21] R. P. Joshi and K. H. Schoenbach, Phys. Rev. E 62, 1025 (2000).

[22] R. P. Joshi, Q. Hu, R. Aly, K. H. Schoenbach, and H. P. Hjalmarson, Phys. Rev. E 64, 011913 (2001).

[23] J. C. Weaver, Ann. N.Y. Acad. Sci. 720, 141 (1994).

[24] J. C. Neu and W. Krassowska, Phys. Rev. E 59, 3471 (1999).

[25] J. Teissie and M. P. Rols, Ann. N.Y. Acad. Sci. 720, 98 (1994).

[26] R. W. Glaser, S. L. Leikin, L. V. Chernomordik, V. F. Pastushenko, and A. I. Sokirko, Biochim. Biophys. Acta 940, 275 (1988).

[27] M. Winterhalter and W. Helfrich, Phys. Rev. A 36, 5874 (1987).

[28] W. Meier, A. Graff, A. Diederich, and M. Winterhalter, Phys. 
Chem. Chem. Phys. 2, 4559 (2000).

[29] V. A. Parsegian, Nature (London) 221, 844 (1969).

[30] J. C. Weaver, R. A. Mintzer, H. Ling, and S. R. Sloan, Bioelectrochem. Bioenerg. 15, 229 (1986).

[31] S. E. Feller and R. W. Pastor, Biophys. J. 71, 1350 (1996).

[32] S. I. Sukharev, P. Blount, B. Martinac, F. R. Blattner, and C. Kung, Nature (London) 368, 265 (1994).

[33] T. Benachir and M. Lafleur, Biophys. J. 70, 831 (1996).

[34] S. R. James, R. A. Demel, and C. P. Downes, Biochem. J. 298, 499 (1994).

[35] H. Isambert, Phys. Rev. Lett. 80, 3404 (1998).
[36] S. H. White, Biophys. J. 14, 155 (1974).

[37] D. V. Zhelev and D. Needham, Biomembranes 1147, 89 (1993).

[38] O. Sandre, L. Mareaux, and F. Brochard-Wyart, Proc. Natl. Acad. Sci. U.S.A. 96, 10591 (1999).

[39] D. C. Chang and T. S. Reese, Biophys. J. 58, 1 (1990).

[40] F. Brochard-Wyart, P. G. de Gennes, and O. Sandre, Physica A 278, 32 (2000).

[41] E. M. Renkin, J. Gen. Physiol. 38, 225 (1954).

[42] J. Israelachvili, Intermolecular and Surface Forces, 2nd ed. (Academic, London, 1992). 